\title{
Effect of feldspathic porcelain application on the marginal discrepancy of zirconia and titanium complete-arch fixed implant-supported frameworks
}

Burak YILMAZ, ${ }^{a}$ Faris A. ALSHAHRANI, ${ }^{b}$ Ediz KALE, ${ }^{c}$ and William M. JOHNSTON ${ }^{d}$

a Department of Restorative Science and Prosthodontics, The Ohio State University College of Dentistry, USA.

b University of Dammam, College of Dentistry, Department of Substitutive Dental Sciences, Saudi Arabia.

c Department of Prosthodontics, Mustafa Kemal University Faculty of Dentistry, Turkey.

a Division of General Practice and Materials Science, The Ohio State University College of Dentistry, USA

\section{Background and Aim}

Veneering with porcelain may adversely affect the marginal fit of longspan computer-aided design and computer-aided manufacturing (CAD/CAM) implant-supported fixed prostheses. Moreover, data regarding the precision of fit of CAD/CAM-fabricated implant-supported complete zirconia fixed dental prostheses (FDPs) before and after porcelain layering is limited.

The purpose of this study was to evaluate the effect of porcelain layering on the marginal fit of CAD/CAM-fabricated complete-arch implantsupported screw-retained FDP with presintered zirconia framework comparing to titanium.

\section{Methods and Materials}

An autopolymerizing acrylic resin fixed complete denture framework prototype was fabricated on an edentulous training typodont master model (all-on-4 concept; Nobel Biocare) with 2 straight in the anterior and 2 distally tilted internal-hex dental implants in the posterior with multi-unit abutments bilaterally in canine and first molar teeth locations. A 3dimensional (3D) laser scanner (S600 ARTI, Zirkonzahn) was used to digitize the prototype and the master model using scan bodies to generate a virtual 3D CAD framework.

Five presintered zirconia (ICE Zirkon Translucent - 95H16, Zirkonzahn) and 5 titanium (Titan 5 - 95H14, Zirkonzahn) frameworks were fabricated using the CAM milling unit (M1 Wet Heavy Metal Milling Unit,

Zirkonzahn)

The one-screw test was applied by fixing the frameworks at the location of maxillary left first molar abutment and an industrial CT scanner (XTH 225 - Basic Configuration; Nikon) was used to scan the framework-model complex to check the passive-fit of the frameworks on the master model. The scanned data were transported in surface tessellation language (STL) from Volume Graphics analysis software to PolyWorks analysis software using Maximum-fit algorithm to fit scanned planes in order to mimic the mating surfaces in the best way. 3D virtual assessment of the marginal fit was performed at the abutment-framework interface at the maxillary right canine (Gap 3) and right first molar (Gap 4) abutments without prosthetic screws.

Facial/buccal aspects of the teeth on frameworks were layered with corresponding porcelain (Initial - Dental Ceramic System; GC) and CT scanned again using the same protocol.

Marginal fit measurements were made for 4 groups; titanium (Ti) (control), porcelain-layered titanium (Ti-P) (control), zirconia ( $\mathrm{Zr}$ ), and porcelain-layered zirconia ( $\mathrm{Zr}-\mathrm{P})$. 3D discrepancy mean values were computed and calculated, and the results were analyzed with a repeated measures 3-way ANOVA using the maximum likelihood estimation method and Bonferroni adjustments to selected pairwise comparison ttests $(\alpha=.05)$.

\section{Results}

The 3D fit measurements between the circular mating surfaces of the frameworks and implant abutments were obtained for Gap 3 and Gap 4. The ANOVA indicated no statistically significant effect of the gap $(P \geq .075)$ but did indicate a statistically significant interaction between the material and the porcelain layering $(P<.001)$.

The mean discrepancies for both Gaps 3 and 4 were 88, 71, 89 and 175 m respectively for Groups Ti, Ti-P, Zr and Zr-P.

Statistically significant differences were found between Groups $\mathrm{Zr}$ and $\mathrm{Zr}$ $\mathrm{P}$ and between Groups Ti-P and $\mathrm{Zr}-\mathrm{P}(P<.001)$, but no such difference was found between Groups Ti and Ti-P $(P=.768)$.

\section{Conclusions}

Within the limitations of this in vitro study, the following conclusions were drawn:

- Absolute passive fit was not achieved at any stage of fabrication for the CAD-CAM-fabricated complete-arch implant-supported screw-retained FDPs with presintered zirconia or titanium frameworks.

- Porcelain layering had insignificant effect on the 3D marginal integrity of CAD-CAM-fabricated titanium complete-arch implant-supported screw-retained FDP frameworks, however, the effect of porcelain layering on the marginal fit of partially sintered zirconia framework was significant.

- Three-dimensional mean marginal discrepancy values for all groups were within the clinically acceptable limits $(<120 \mu \mathrm{m})$ except for the porcelain layered zirconia frameworks.
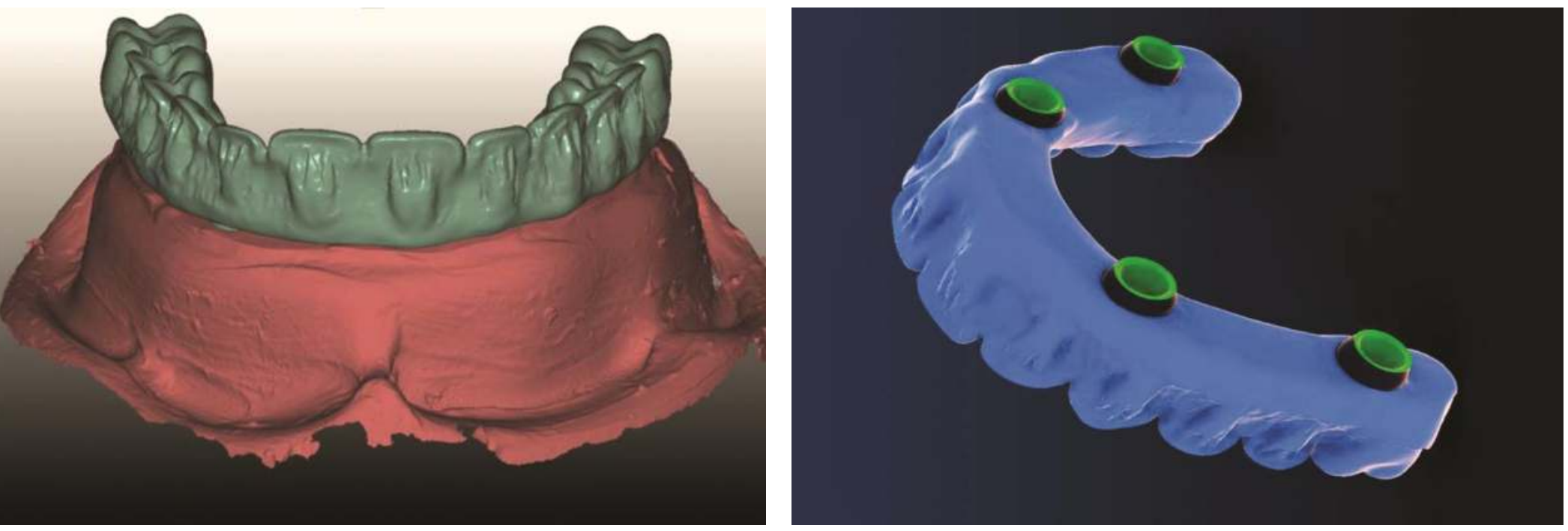

Figure 1.

Figure 2.

Figures 1 through 6 show the applied procedures during the study sequence.

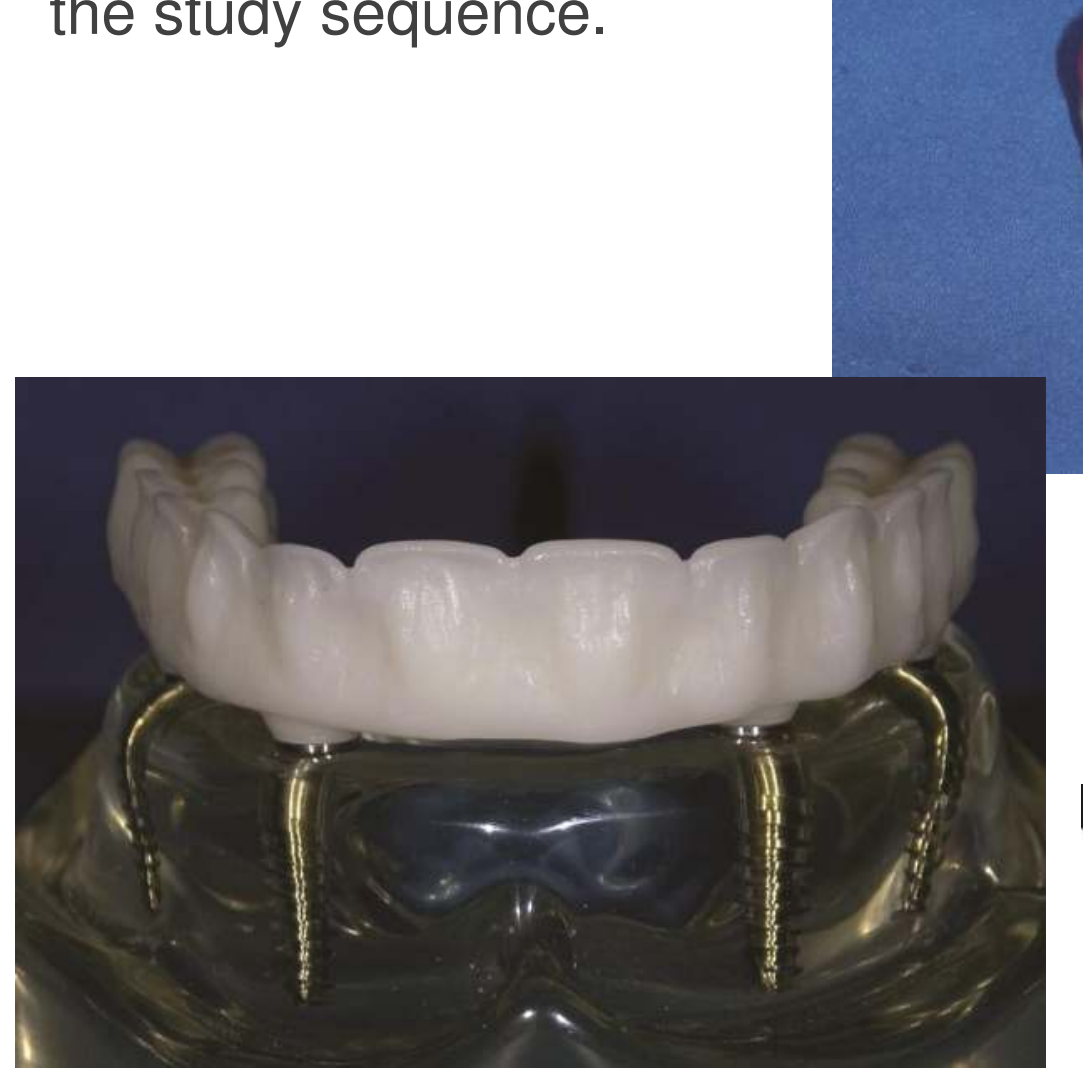

Figure 3.

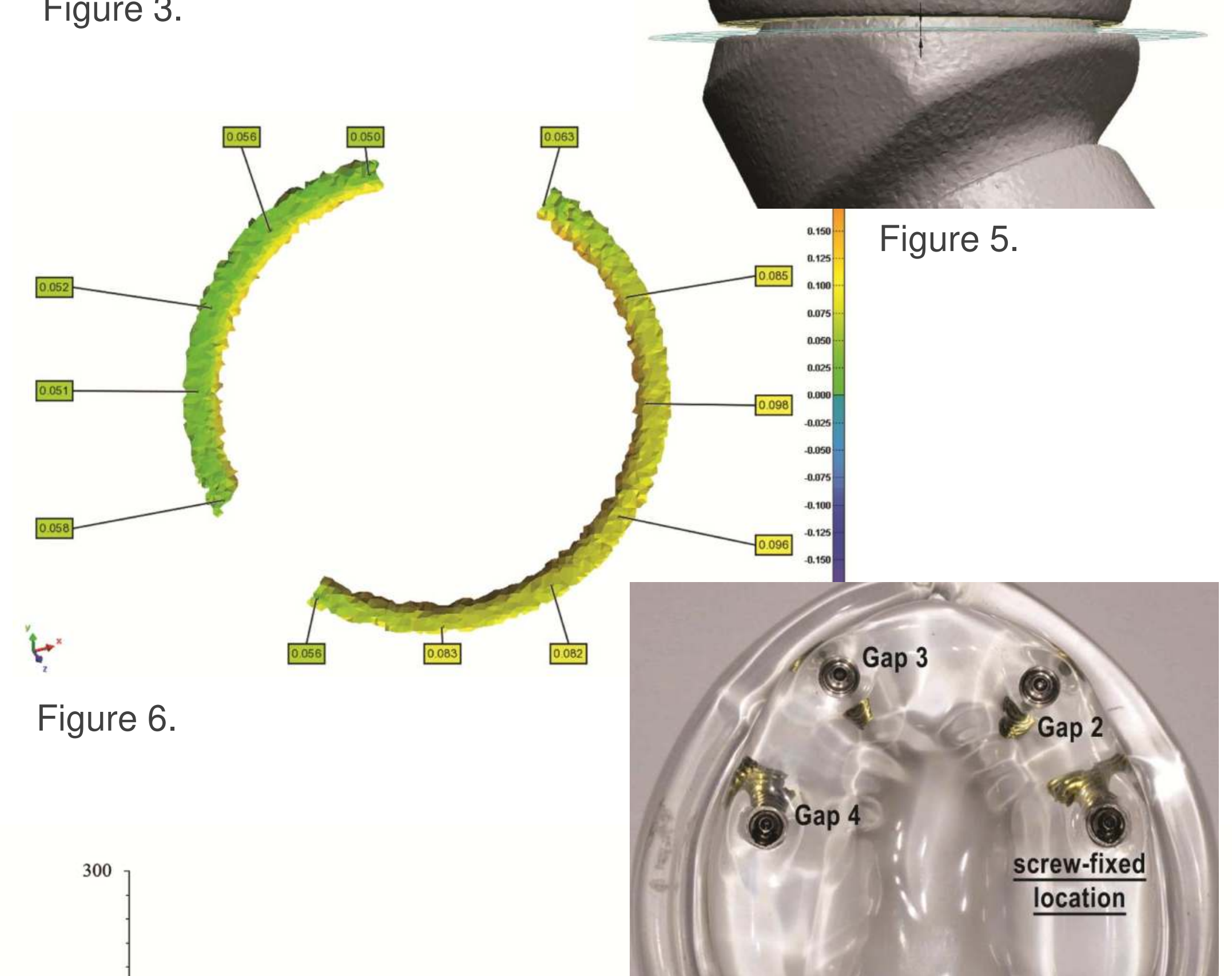

Figure 7

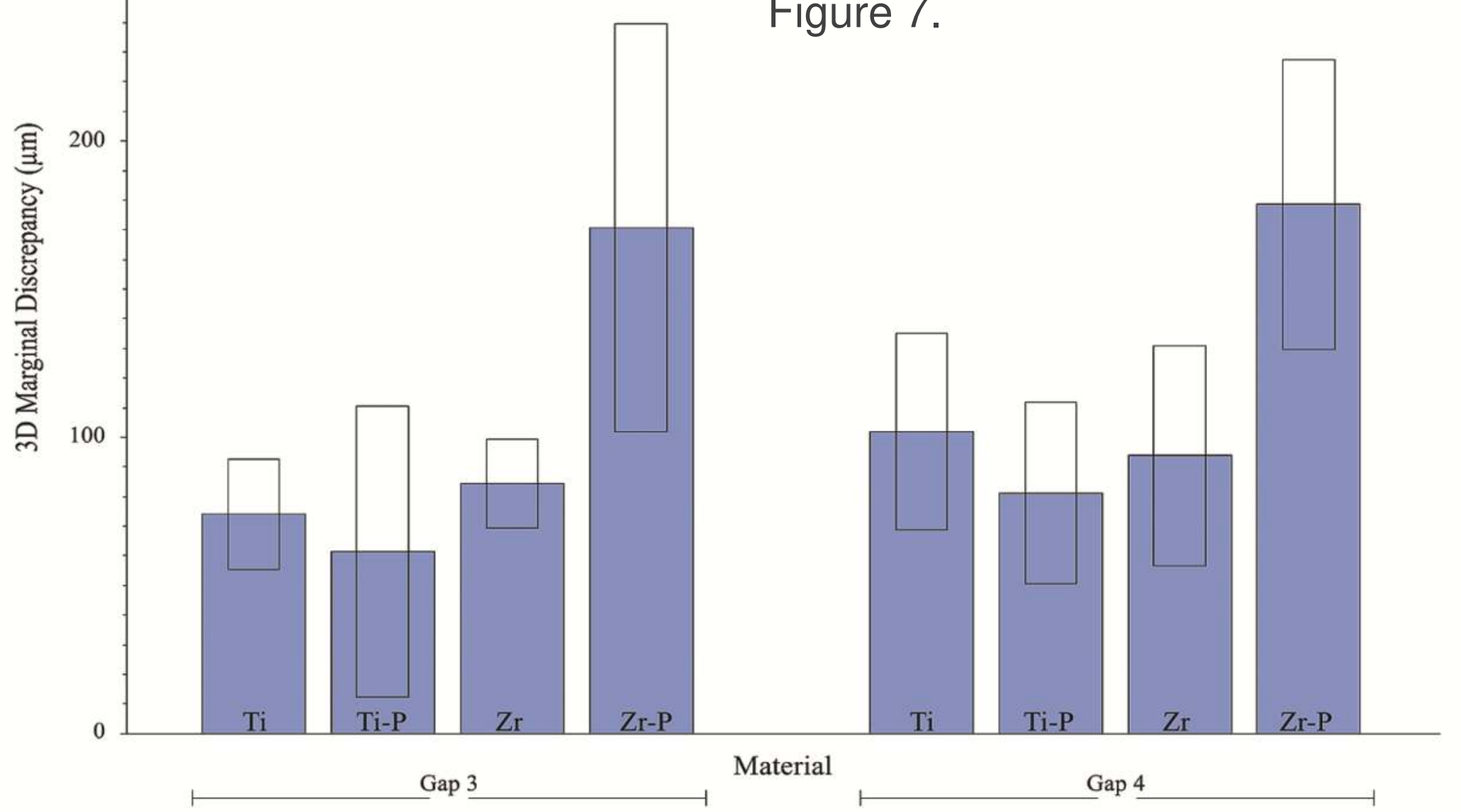

References

1. Osseointegration and its experimental back ground. J Prosthet Dent 1983:50:399-410 2. The significance of passive framework fit in implant prosthodontics: current status. Implan The significance of pas
Dent 2001;10:85-92

3. Precision of fit of implant-supported screw retained 10-unit computer-aided-designed and computer-aided- manufactured frameworks made from zirconium dioxide and titanium: an in vitro study. Clin Oral Implants Res 2014;25:165-74

4. Failures and complications in 391 consecutively inserted fixed prostheses supported by Brånemark implants in edentulous jaws: a study of treatment from the time of prosthesis placement to the fist end in vitro precision of Oral Implants Res 2015:26:44-9.

The estimation of cement film thickness by an in vivo technique. Br Dent J 1971:131:107-11 